\title{
Stereotactic Core Biopsy Following Screening Mammography: A Danish Retrospective National Cohort Study
}

\author{
Søren Redsted ${ }^{*}$, Quynh T. H. Nguyen², René Depont Christensen ${ }^{3}$, Grethe Myrtue", \\ Tina Di Caterino5, Marianne Djernes Lautrup ${ }^{6}$ \\ ${ }^{1}$ Department of Radiology, Aarhus University Hospital, Aarhus, Denmark \\ ${ }^{2}$ Department of Plastic Surgery, Odense University Hospital, Odense, Denmark \\ ${ }^{3}$ Research Unit of General Practice, University of Southern Denmark, Odense, Denmark \\ ${ }^{4}$ Department of Radiology \& Mammography, SLB Vejle Hospital, Vejle, Denmark \\ ${ }^{5}$ Department of Pathology, SVS Esbjerg Finsensgade, Esbjerg, Denmark \\ ${ }^{6}$ Department of Breast Surgery, SLB Vejle Hospital, Vejle, Denmark \\ Email: *soered@rm.dk
}

How to cite this paper: Redsted, S. Nguyen, Q.T.H., Christensen, R.D., Myrtue, G., Di Caterino, T. and Lautrup, M.D. (2018) Stereotactic Core Biopsy Following Screening Mammography: A Danish Retrospective National Cohort Study. International Journal of Clinical Medicine, 9, 341-355.

https://doi.org/10.4236/ijcm.2018.95030

Received: February 22, 2018

Accepted: May 5, 2018

Published: May 8, 2018

Copyright $\odot 2018$ by authors and Scientific Research Publishing Inc. This work is licensed under the Creative Commons Attribution International License (CC BY 4.0).

http://creativecommons.org/licenses/by/4.0/ (c) (i) Open Access

\begin{abstract}
Background: Since introducing stereotactic core biopsy (SCB) on breast lesions in Denmark, no national follow-up of the procedure has been executed. Purpose: To evaluate performance of SCB in Danish mammography screening. 3 areas were selected for evaluation: diagnostic value of SCB, performance of the Danish 7-tier mamma-radiological classifications system, DKBI-RADS, and diagnostic delay for SCB-diagnosis. Materials \& Methods: Danish retrospective national cohort study including 2195 screening patients undergoing SCB. Study period: 01.01.2010 to 30.09.2012. Patients were identified from The Danish National Patient Register. Pathology-data were obtained from the Danish Pathology Database. Radiological-data according to DKBI-RADS were recorded. Diagnostic delay from clinical mammography until diagnosis was registered. Results: 173 SCBs indicated cancer; all operated with 3 cases finalized as benign. 1296 cases were determined benign with diagnostic surgery in 81 cases of which 31 were concluded pre-malignant/malignant. Correlation between DKBI-RADS and pathology diagnosis: 329 of 485 DKBI-RADS3, 227 of 450 DKBI-RADS4 were benign. 4 of 16 DKBI-RADS5 were benign. The diagnostic value of pre-malignant/malignant SCB related to results from surgery showed $94.4 \%$ sensitivity and a positive predictive value of $93.9 \%$. Median diagnostic-time of single-biopsy was 13 days. Conclusion: The performance of SCB in Denmark is comparable to international studies regarding the diagnostic value of malignant SCB. The study indicates that DKBI-RADS classifications are not used consistently regarding micro-calcifications selected in screen-
\end{abstract}


ing-mammographies. Diagnostic delay is acceptable, subject to EUSOMA specifications, regarding single-biopsy.

\section{Keywords}

Stereotactic Core Biopsy, Breast Cancer, Micro Calcification, 7-Tier

Classification, Screening Mammography, Diagnostic Delay

\section{Introduction}

Breast cancer is the most common cancer among women in Denmark with about 4800 new cases every year and with an incidence rate increasing steadily for years [1] [2]. The increase in breast cancer awareness and national use of screening mammography has led to early detection of pre-malignant and early stage non-palpable breast cancer. In Denmark, women between ages 50 - 69 are invited to a screening mammography bi-annually, resulting in approx. 500,000 screening mammographies annually [3]. Of these $2.6 \%$ are invited to additional mammography and ultrasound (US) [3]. Approximately $65 \%$ invited for further examination do not have cancer or pre-malignancy [3]. Most of the women will be definitively, diagnostically clarified with US-guided core biopsies.

Some lesions detected on screening mammography cannot be verified by US examination, typically micro-calcifications. Thus to determine these, a core biopsy for pathological examination is required. The sampling technology stereotactic core biopsy (SCB) provides a method to verify the diagnosis [4] [5].

\section{Materials and Method}

\subsection{Population}

A cohort from the Danish National Patient Register ${ }^{1}$ was defined by identifying all women registered with the procedure codeKTHA10A of SCB within 90 days after a screening mammography (UXRC45) between 1st January 2010 and 1st October 2012.

Figure 1 shows a flow chart of the study population.2195 women were selected for SCB. However, the procedure-code for SCB was registered but SCB not carried out in 46 cases. Registrations do not indicate whether procedure was merely cancelled due to for example no show. Altogether, 2288 SCB were performed on 2149 women. Bilateral SCBs were performed on 58 women and re-SCBs on 51 women.

\footnotetext{
${ }^{1}$ Denmark has several high-quality national registers including the Danish Civil Registration System (CPR Register). Every person is registered with a national, social security number (CPR number) indicating time of birth and gender. Death, emigration, residence and several other data is also registered under the CPR registration. In the Danish public health system, all patients treated in a hospital are registered in The National Patient Register (NPR) with their CPR number, a code of diagnosis and a code of treatment supplied as well as a code of the hospital and the department treating the patient. The pathologists register the histological information about all biopsies and specimens in The National Pathology Data Bank (Patobank).
} 


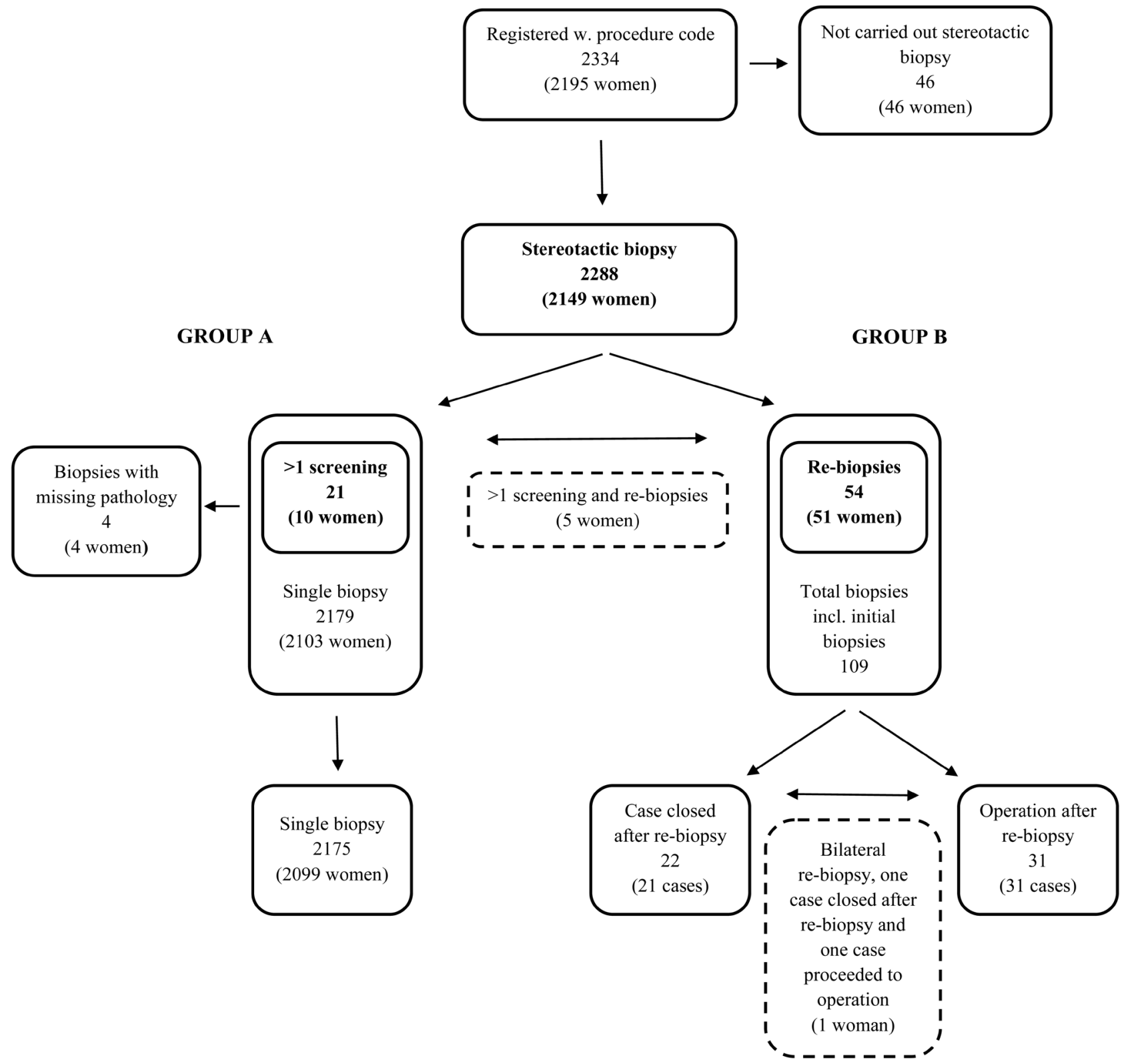

Figure 1. Flow chart showing the study population.

The study group was divided into two sub-cohort groups:

- Group A, single SCBs group, which includes women (2103) undergoing clinical mammography, SCB and in some cases operation in order to diagnose or treat pre-malignant or malignant lesions. The group also includes women (10) with more than one screening during the study period. Four women were excluded because of missing pathology information. In total, 2175 single SCBs performed on 2099 women were included in the single SCB sub-cohort.

- Group B, includes women (51) undergoing re-SCBs defined as SCBs performed within 90 days from the first SCB. 109 SCBs were performed of which 54 were re-SCBs. Note that in the flow-chart we reference cases, since one 
woman was included in both groups because of bilateral SCB; one case was closed after re-SCB; and one case proceeded to operation. In total 21 cases were finalized after re-SCB and 31 cases underwent an operation after an additional SCB. Finally, five women with more than one screening in the study period and re-SCBs were included. One woman underwent a second re-biopsy. The initial and additional SCB was performed on both breasts. The woman was counted in both sub-groups.

The analyses concerning the correlation between DKBI-RADS and final pathology result (single-SCB, re-SCB or surgery) include single-SCB as well as re-SCB.

\subsection{Mammography and Radiological Classification}

The European and in particular the northern European countries, including the UK, but also Australia and New Zealand, use a 5-tier classification system. These are typically either modified, versions of the American BI-RADS classifications, or a 5-tier classification developed by The Royal College of Radiologists Breast Group, or a Tabar 5-tier classification [6] [7] [8]. The Danish classification system (DKBI-RADS), as recommended by DBCG [1], is a modified classification, based on a combination of versions of the American BI-RADS and possibly the Tabar classification. To clarify the difference both the current American BI-RADS and DKBI-RADS classifications are illustrated below (Figure 2 and Figure 3).

All Danish mammography centres provided pathology reports. All except the largest centre made clinical mammographies, in total 1181, available for the study. These were reviewed and mammography descriptions with missing classifications were classified subject to the following methodology: If the mammography was described with suspicious or malignant characteristics, the lesions were considered suspicious or malignant. Such mammographic findings were classified as DK-B4 if suspicious and DK-B5 if malignant. Similarly, a large part of mammographies with most likely benign morphological characteristics, lesions with non-specific morphology or with no further morphological description were considered DK-B3.

Patients in Denmark are referred to the radiology breast-unit for a clinical mammography. This includes supplementary mammography combined with a clinical examination, ultrasound-scan of breast and axilla and if relevant, biopsies. Additional MRI of the breasts is available if necessary. Triple diagnostics form the basis for Danish breast-diagnostics, and comprise palpation, diagnostic imaging, and fine needle or core needle biopsy. Lesions, which are non-detectable by clinical-mammography, predominantly micro-calcifications and a minute group of small lesions without calcification, cannot be appropriately diagnosed with triple diagnostics and are referred to SCB.

We know that about $30 \%$ of screening [9] selected micro-calcifications are pre-malignant or malignant and should according to DKBI-RADS, be classified as DK-B4. However, the classification DK-B3 is frequently used to allow for 


\section{American BI-RAD classification of mammographic lesions}

\begin{tabular}{|c|c|c|c|}
\hline $\begin{array}{l}\text { BI-RAD } \\
\text { Class }\end{array}$ & Description & $\begin{array}{c}\text { Probability of } \\
\text { Malignancy (\%) }\end{array}$ & Follow-up \\
\hline $\mathbf{0}$ & Needs additional evaluation & & $\begin{array}{l}\text { Diagnostic mammogram, } \\
\text { ultrasonographic image }\end{array}$ \\
\hline 1 & Normal mammogram & 0 & Yearly screening \\
\hline 2 & Benign lesion & 0 & Yearly screening \\
\hline 3 & Probably benign lesion & $<2$ & Short interval follow-up \\
\hline $4 *$ & Suspicious for malignancy & 20 & Biopsy \\
\hline 5 & Highly suspicious for malignancy & 90 & Biopsy \\
\hline 6 & Biopsy proven malignancy & 100 & Treatment \\
\hline
\end{tabular}

*the ACR recommends that each site be divided into three subcategories: 4A, low suspicion; 4B, intermediate suspicion; and 4C, moderate concern but not classic for malignancy.

Source for BI-RAD classification: ACR/cancernetwork.com

\section{Danish modified BI-RAD ( DKBI-RAD) classification of mammographic lesions}

\begin{tabular}{|c|c|c|c|}
\hline $\begin{array}{l}\text { DK BI-RAD } \\
\text { Class }\end{array}$ & Description & $\begin{array}{c}\text { Probability of } \\
\text { Malignancy }(\%)\end{array}$ & Follow-up \\
\hline DK B0 & Needs additional evaluation & & \\
\hline DK B1 & Normal & & None \\
\hline DK B2 & Benign & & $\begin{array}{l}\text { None. If palpable or } \\
\text { ultrasound mass then possibly } \\
\text { biopsy }\end{array}$ \\
\hline DK B3 & Undecided/atypical/indifferent & & Biopsy \\
\hline DK B4* & Malignancy suspect & & Biopsy \\
\hline DK B5 & Malignant & & Biopsy \\
\hline DK B6 & Biopsy verified malignant & & Treatment \\
\hline \multicolumn{4}{|c|}{ *Unlike the American BI-RAD class 4, the Danish DK B4 has no sub-classes. } \\
\hline
\end{tabular}

Figure 2. The Danish modified BI-RADS and the American BI-RADS.

biopsies without forcing surgery before deemed absolutely necessary. A DK-B3 diagnosis indicates that the breast radiologist retains responsibility for completing diagnosis through biopsies until a malignancy suspicion is verified as malignant or benign. If classified as DK-B4, then said responsibility for verifying diagnosis is commonly transferred to the surgeon and diagnostic surgery in the Danish diagnostic system. For ease of reference, the process is shown below.

\subsection{Stereotactic Core Biopsy and Equipment in Denmark}

SCB makes use of the underlying principle of parallax in order to determine the depth or "Z-dimension" of the target lesion and thereby measure the targeted lesion tri-dimensionally [10] [11].

SCB is carried out in several mammography centres in Denmark. As shown in 


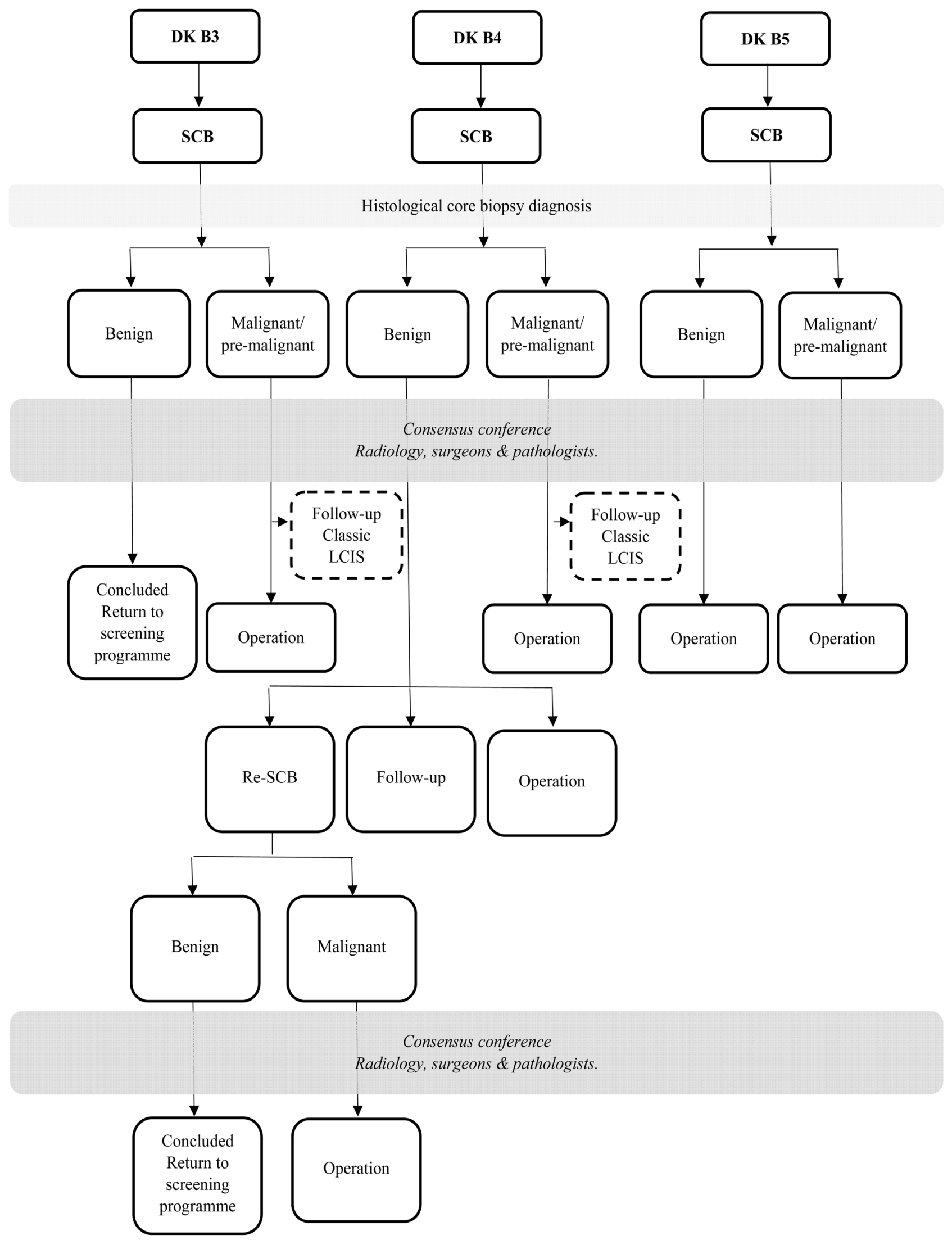

Figure 3. Pathway. 
Table 1, there is some level of centralization of the stereotactic procedure. Table 1 shows that the various centres use a variety of stereotactic equipment both vertical (add-on) and horizontal. Also, SCB systems in use vary, with pros and cons for each of these systems [12]. Further analysis of this aspect is, however, outside the scope of this article.

\subsection{Pathological Classification}

We collected information of pathological diagnosis from SCBs and surgical excisions from the Danish national register of pathology, Patobank. Dates of SCB, surgery and verified diagnosis were registered.

The pathological findings were classified as benign, pre-malignant or malignant according to the Danish national guidelines [1].

If micro-calcifications were the reason for SCB but SCB did not identify these, the SCB was considered non-representative.

Benign lesions include a variety of benign morphologies.

Atypical lesion is a heterogeneous group of histological findings. The most frequent diagnosis was radial scar and intraductal papilloma (IP) but also included Flat Epithelia Atypia (FEA) and Atypical Ductal Hyperplasia (ADH). Lobular carcinoma in situ (LCIS) was classified separately. Complete excision of the two first mentioned diagnoses is recommended. We classified all IP as pre-malignant lesions since they, according to the guidelines, should be removed, while no specific recommendations exist regarding FEA and ADH. These lesions may co-exist with malignancies or pre-malignancies. LCIS was registered as a pre-malignant lesion since LCIS is associated with increased lifetime risk of subsequent malignancy [13] [14].

Table 1. Overview of stereotactic equipment in screening centres in Denmark, 2010-2012.

\begin{tabular}{|c|c|c|}
\hline Centre & Stereotactic System & Biopsy System \\
\hline Aabenraa Hospital & Novation Add-ON & $\begin{array}{l}\text { Vacore Bard, } \\
\text { single GN-biopsy 10G }\end{array}$ \\
\hline Aalborg University Hospital & Hologic horizontal & ATEC Pearl Vacuum System \\
\hline Aarhus University Hospital & $\begin{array}{l}\text { Mammotest Siemens } \\
\text { (Fischer) horizontal }\end{array}$ & ATEC Pearl Vacuum System \\
\hline Esbjerg Hospital & Novation Add-ON & $\begin{array}{c}\text { Mammotome Vacuum System } \\
\text { and angiotech GN-Biopsy }\end{array}$ \\
\hline Herlev Hospital & Novation Add-ON & $\begin{array}{l}\text { Vacore Bard, } \\
\text { single GN-biopsy 10G }\end{array}$ \\
\hline Odense University Hospital & $\begin{array}{l}\text { Mammotest Siemens } \\
\text { (Fischer) horizontal }\end{array}$ & $\begin{array}{c}\text { Vacore Bard, } \\
\text { single GN-biopsy 10G }\end{array}$ \\
\hline Ringsted Hospital & Hologic horizontal & $\begin{array}{l}\text { Mammotome, Bard single } \\
\text { GN-Biopsy }\end{array}$ \\
\hline Rigshospitalet & Novation Add-ON & $\begin{array}{c}\text { Bard single GN-biopsy; Vacore } \\
\text { Bard, single GN-biopsy 10G }\end{array}$ \\
\hline Vendsyssel Hospital & Novation Add-ON & Full core, Bard, GN-biopsy \\
\hline
\end{tabular}


Pre-malignant lesions comprise Ductal Carcinoma in Situ (DCIS) and Pleomorphic Lobular Carcinoma in Situ PLCIS, or findings suspicious of malignancy but not conclusive in the SCB material. The majority of malignant lesions comprise Invasive Ductal Carcinoma (IDC) and Invasive Lobular Carcinoma (ILC).

\section{Statistical Analysis}

Descriptive statistics and comparative analyses were used. Categorical variables were summarized by frequencies and percentages, continuous variables by mean, standard deviation, minimum, maximum, and the quartile set. The data sources were linked by pseudo-anonymized patient id and breast location (left/right). Re-SCBs were defined as SCBs in the same breast within 90 days. For time to final diagnostic clarification, we considered the time from mammography to SCB or operation. Pre-malignant and malignant SCBs were considered final since subsequent surgery per definition has a curative intention. All statistical programming and analyses were performed using STATA 14.0 (Stata Corp. College Station, TX, USA).

\section{Results}

\subsection{Pathological Result for Group a Single SCB (Table 2)}

A comparison of SCB pathological findings and subsequent surgical excision if performed is shown in Table 3. In total 899 (41\%) of 2175 single SCBs were referred to surgery. 81 (6\%) benign SCB were operated for further diagnosis and of these $38 \%$ were found to be malignant. 26 (45\%) with non-representative SCBs also underwent diagnostic surgery without obtaining a diagnosis from a re-SCB. 619 of the 647 premalignant lesions (96\%) were operated according to guidelines. All 173 malignant lesions were operated. In three cases (2\%) no malignancy was found.

Table 2. Pathological results of single SCBs.

\begin{tabular}{cc}
\hline SCB & Number (\%) \\
\hline Benign & $1296(59)$ \\
Pre-malignant & $648(30)$ \\
Malignant & $173(8)$ \\
Not representative & $58(3)$ \\
Total & $2175(100)$ \\
\hline
\end{tabular}

Table 3. Final pathological results of operation performed on $899 / 2175$ single SCBs (\%).

\begin{tabular}{|c|c|c|c|c|}
\hline SCB diagnosis & Benign (\%) & Pre-malignant (\%) & Malignant (\%) & Total (\%) \\
\hline Benign [1296] & $50(62)$ & $20(24)$ & $11(14)$ & $81(100)$ \\
\hline Pre-malignant [647] & $45(7)$ & $464(75)$ & $110(18)$ & $619(100)$ \\
\hline Malignant [173] & $3(2)$ & $19(11)$ & $151(87)$ & $173(100)$ \\
\hline Not repres. [58] & $13(50)$ & $9(35)$ & $4(15)$ & $26(100)$ \\
\hline Total [2175] & $111(12)$ & $513(57)$ & $276(31)$ & $899(100)$ \\
\hline
\end{tabular}




\subsection{The Diagnostic Value of a Pre-Malignant or Malignant Group A SCB}

The diagnostic value of pre-malignant/malignant SCB in relation to the end result from surgery shows $94.4 \%$ sensitivity and a positive predictive value of 93.9\% (Table 4).

\subsection{Pre-Malignancies of Group A Single SCB}

Specifications of pre-malignancies are illustrated in Table 5. In 13/619(2\%) SCBs the diagnosis was not specific as to whether it was DCIS, LCIS or atypical lesions. All underwent diagnostic operation.

The majority of pre-malignancies were identified as DCIS in 567 (91\%) SCBs. Isolated LCIS was found in 15 (2\%) SCBs. Pre-malignant atypia was identified in $52(8 \%)$ SCBs and operation was performed in 43 (83\%) cases.

\subsection{Pathological Result of Re-SCBs (Group B)}

Re-SCBs performed on 51 women. Two women were included in more than one screening and there were bilateral re-SCBs. In total, 54 SCBs were performed on 51 women.

Table 4. Diagnostic value of a malignant or pre-malignant SCB.

\begin{tabular}{|c|c|c|c|c|}
\hline \multicolumn{5}{|c|}{ SCB result } \\
\hline Surgery result & Mal/Pre-mal & Benign & Non-representative & Total \\
\hline Mal/Pre-mal & $744(93.9 \%)$ & $31(38.3 \%)$ & $13(50 \%)$ & 788 \\
\hline Benign & $48(6.1 \%)$ & $50(61.7 \%)$ & $13(50 \%)$ & 111 \\
\hline Total & 792 & 81 & 26 & 899 \\
\hline \multicolumn{5}{|c|}{ [95\% Confidence Interval] } \\
\hline Prevalence & $\operatorname{Pr}(\mathrm{A})$ & $88 \%$ & $85 \%$ & $89.7 \%$ \\
\hline Sensitivity & $\operatorname{Pr}(+\mid A)$ & $94.4 \%$ & $92.6 \%$ & $95.9 \%$ \\
\hline Specificity & $\operatorname{Pr}(-\mid N)$ & $56.8 \%$ & $47 \%$ & $66.1 \%$ \\
\hline Positive predictive value & $\operatorname{Pr}(\mathrm{A} \mid+)$ & $93.9 \%$ & $92 \%$ & $95.5 \%$ \\
\hline
\end{tabular}

Table 5. Pre-malignant finding of single SCB that was operated (\%).

\begin{tabular}{ccccc}
\hline SCB diagnosis & Benign (\%) & Pre-malignant () & Malignant (\%) & Total (\%) \\
\hline DCIS [567] & $32(6)$ & $426(77)$ & $98(18)$ & $556(100)$ \\
LCIS [15] & $2(29)$ & $2(29)$ & $3(43)$ & $7(100)$ \\
Atypia [52] & $9(21)$ & $29(67)$ & $5(12)$ & $43(100)$ \\
No evaluation [13] & $2(15)$ & $7(54)$ & $4(31)$ & $13(100)$ \\
Total [647] & $45(7)$ & $464(75)$ & $110(18)$ & $619(100)$ \\
\hline
\end{tabular}


31 of the re-SCB cases proceeded to surgery. 22 of 31 benign re-SCBs were accepted as benign after re-SCB. 9 benign re-SCBs were still not accepted as benign and proceeded to operation. 22 re-SCBs were diagnosed as either premalignant (18) or malignant (4) and were operated according to guidelines.

\subsection{Comparison between DK BI-RADS Classification and the Final Pathological Diagnosis}

Table 6 is an overview of the lesions assigned to the DKBI-RADS categories (DK-B3, DK-B4 and DK-B5) and the distribution of the final pathology-result of the lesion, irrespective of whether the final result was reached after single-SCB, re-SCB or surgery according to each DKBI-RADS category. Earlier we described 969 mammographies with DKBI-RADS classification; four women were excluded because of missing pathology, five women were included in both sub-cohort groups of which two women underwent re-SCB, bilaterally. In total, the analyses included 951 cases where classification was possible.

\section{Diagnostic Delay}

To examine potential delays, we evaluated the process from clinical mammography to definitive diagnosis by either SCB or surgical excision. Throughout the study we have divided the population into two subgroups: the sub-cohort groups A: single SCB and B: re-SCB respectively.

There was inconsistency between the number of mammographies and pathology evaluations due to lack of mammography descriptions, lack of DKBI-RADS classification and in a few cases lack of pathology diagnosis. Half the 805 women undergoing single SCB were diagnosed within 13 days. The group of re-SCBs included 21 cases, and half of the patients were diagnosed within 38 days (Table 7 and Table 8). 75\% of the women undergoing single SCB were diagnosed within 27 days but it took about twice as long (52 days) to diagnose the sub-cohort group of re-SCB.

The meantime of the single SCB and the re-SCB sub-groups was 18 and 40 days, respectively.

Table 6. Final pathological results (\%).

\begin{tabular}{ccccc}
\hline DK BI-RADS (B) & Benign (\%) & Pre-malignant (\%) & Malignant (\%) & Total (\%) \\
\hline DK B3 & $329(68)$ & $113(23)$ & $43(9)$ & $485(100)$ \\
DK B4 & $227(50)$ & $149(33)$ & $74(17)$ & $450(100)$ \\
DK B5 & $4(25)$ & $6(37)$ & $6(38)$ & $16(100)$ \\
Total & $560(59)$ & $268(28)$ & $123(13)$ & $951(100)$ \\
\hline
\end{tabular}

Table 7. Time spent on diagnosis process of single SCB.

\begin{tabular}{ccccccc}
\hline Variable & N & Mean & SD & p25 & p50 & p75 \\
\hline Total time & 805 & 18.46 & 19.94 & 5 & 13 & 27 \\
\hline
\end{tabular}


Table 8. Time spent on diagnosis process of re-SCB.

\begin{tabular}{ccccccc}
\hline Variable & N & Mean & SD & p25 & p50 & p75 \\
\hline Total time & 21 & 39.86 & 19.41 & 28 & 38 & 52 \\
\hline
\end{tabular}

\section{Discussion}

A strength of this study is that it is based on a large homogeneous cohort from the Danish screening mammography population. Denmark has very complete national registers, allowing us to avoid selection bias and, furthermore, everyone has a civil registration number providing access to information for each patient.

Collection of data was carried out by one person to ensure a uniform database. Finally, it is a large national study, which includes all centres in Denmark in terms of pathology reports.

The study has limitations, too. Unfortunately, the clinical mammographyreports are missing from one centre, reducing statistical power as regards DKBI-RADS classifications. Approximately $1 / 3$ of the mammography reports did not include DKBI-RADS classifications and were classified based on descriptions.

The centres use different equipment. The volume of SCB specimens may be associated with failure to obtain correct histological diagnosis [12]. It is anticipated that types of equipment may influence the performance of SCB. Whilst the focus of this study is on the performance of SCB as part of the Danish screening mammography, and not on a qualitative examination of SCB equipment, another study into this subject is suggested.

In our study, up to $32 \%$ of DK-B3 was diagnosed with malignancy or pre-malignancy.

It is expected that the frequency of malignant lesions for each DKBI-RADS category will not correspond well the current American BI-RADS due to the modified DKBI-RADS. The yield of cancer for American BI-RADS3 is $2 \%$ or fewer; $23 \%-25 \%$ among BI-RADS4 and previously published cancer outcomes of BI-RADS5 was $81 \%-100 \%$ [4] [15] [16]. In addition, it should be mentioned that the recommended cancer risk of $2 \%$ or fewer for American BI-RADS3 are based on follow-up mammographies and not on a biopsy approach [17].

Other studies have reported variation in malignancy rates. Literature suggests a ratio of malignancy among American BI-RADS3 lesions on SCB between 4\% $18 \%$ [18] [19]. One study found, as we did, that half of the American BI-RADS4 lesions were benign [20]. It is important to note that the reason behind diagnostic problems is that screening-detected micro-calcifications often are very discrete and in an early stage. As there is no morphologically clear indication of these, they are difficult to classify.

In respect of the performance of SCB, our study shows concordance of $93.9 \%$ of suspicious SCBs in comparison to final diagnosis, which is comparable to the $80 \%-96 \%$ shown in earlier international studies [9], where the results are based on the use of a variety of biopsy equipment as in our study. We found that $6 \%$ of 
cases with benign SCBs were referred to diagnostic surgery. The reason for referring to surgery and re-SCB may be that there is no consensus in Denmark regarding the strategy for sub-pathological core SCB diagnoses such as FEA, ADH and sub-categories of LCIS. Another reason could be discrepancy between DKBI-RADS classifications and the SCB findings. $2 \%$ of pre-malignant SCBs were not classified as LCIS, DCIS or atypia, which makes re-SCB or surgery inevitable.

In terms of re-SCBs, we found 8 pre-malignant/malignant cases out of 54 cases undergoing re-SCB. A reason could be that these re-SCBs may have been carried out in order to determine the size of the lesion or multi-focality with a view to planning of surgery.

$52 \%$ of non-representative cases were operated for a diagnosis. X-ray of the SCB specimen as part of the SCB procedure serves to confirm that a representative amount of micro-calcification from the index lesion is included in the specimen.

Calcification potentially lost in the course of trimming the paraffin block when preparing $\mathrm{H} \& \mathrm{E}$ sections should not be a cause of discrepancy [21]. However, in Denmark a benign histology report is not accepted without representative micro-calcifications.

$2 \%$ of malignant SCB cases were diagnosed benign after surgery. This may be due to the initial malignant lesion being small and the whole cluster of malignant micro-calcification removed by SCB. Other reasons may be pathologist misinterprets initial SCBs as malignant or surgeon fails to remove correct lesion. What could appear as a misclassification may indeed be caused by one or more of above issues. Hence, the above indicates a potential cause of discrepancy, when operation specimens with $\mathrm{x}$-ray-confirmed calcifications are sent for examination, and the final pathology report, despite thorough examination, is unable to confirm.

Finally, the study intended to clarify whether SCB causes a general diagnostic delay. A diagnostic interval is not only delayed by additional SCB but also prolonged by diagnostic surgical excision in some cases needed to complete diagnosis. All diagnostic tests for breast cancer have known rates of misinterpretation [22], which may cause delay in the diagnostic process. Misjudgement of pathology findings has been reported in $0.7 \%-4 \%$ of breast cancers [23] [24]. Again, this can result in unnecessary assessment including re-SCB or even (diagnostic) operation causing delays.

Generally, delay in any cancer diagnosis is common, and it is accepted that total delay should be as short as possible [25]. European Society of Mastology (EUSOMA) recommends [22] that at least $70 \%$ of patients with non-palpable breast cancer should be diagnosed preoperatively.

According to EUSOMA, time recommendation from diagnosis to final operation is 20 working days, ideally less [22]. We decided only to calculate the delays from mammography to final pathological diagnosis. This could either be after first SCB, re-SCB or diagnostic operation. 
We found, that the median diagnosis time for women with a straightforward clinical examination is acceptable: $75 \%$ of women were finalized within $27 \mathrm{cal}-$ endar days, meeting the recommendation. The expectation is that women undergoing re-SCB would have a delayed diagnostic process, which the study also shows; only $25 \%$ of these women were diagnosed in time (within 28 days).

\section{Conclusions}

This study demonstrates results comparable with other studies as regards malignant SCBs and the final pathology report after surgery [9]. A positive predictive value of $93.9 \%$ on pre-malignant/malignant SCB is a satisfying result, although further study of the falsely negative SCBs would be recommendable in order to obtain a complete test analysis for SCB in Denmark. The number of patients with malignant finds from SCB (38\%) and the $41 \%$, who proceed to surgery from single SCB, appears high and is perhaps also an area for further study in order to determine whether too few SCBs are carried out. Furthermore, it is indicated through this study, that the number of benign SCBs, which have still proceeded to diagnostic surgery, is high and the subsequent number of malignancies found is greater than shown in other studies [9]. This could indicate that there is a need for further centralisation of centres performing SCB in Denmark as well as a study into improving performance regarding the issues identified above.

The DKBI-RADS classification of micro-calcifications is not comparable with other studies. We know from other studies, that approximately $25 \%-30 \%$ of patients selected for SCB have malignant finds and that it is difficult to classify the finds on the basis of mammographies. This is probably the reason why mamma-radiologists in Denmark in general do not want to classify micro-calcification lesions prior to SCB. In part, this may be attributable to the classification being too uncertain as also indicated by many studies. The study indicates that a robust management system is in place in Denmark based on Pathway for Diagnostic Strategies. However, since the DKBI-RADS classification is in reality not a BI-RADS system but a modification hereof and includes elements of other 5-tier classification systems, there are indications in the study to suggest that the Danish BI-RADS reference can lead to confusion. It may therefore be appropriate to change the classification recommendations in Denmark and ensure that the classification system is given a more appropriate name.

Overall, this study found the diagnostic delay to be within the recommendation from EUSOMA for single SCBs. Re-SCBs have a diagnostic delay, which is more than recommended.

\section{Acknowledgements}

The completion of this study was supported by the assistance of co-workers and staff members and their contributions are sincerely appreciated and gratefully acknowledged. 


\section{Funding}

The Region of Southern Denmark has financed postdoctoral research for Marianne Lautrup. Department of Breast Surgery, Lillebaelt Hospital, Vejle has financed the extraction of data from national registers, and the Research Council Lillebaelt Hospital has financed statistical and database set-up assistance.

\section{Conflict of Interest and Ethics}

The authors declare that there is no conflict of interest.

The study has been approved by the Danish Data Protection Agency and the Danish Health and Medicines Authority, Inspection and Patient Security.

\section{References}

[1] Danish Breast Cancer Cooperative Group. DBCG.dk. http://www.dbcg.dk/

[2] Cancer. Cancer.dk. http://www.cancer.dk/

[3] Lynge, E., Bak, M., Euler-Chelpin, M., Kroman, N., Lernevall, A., Mogensen, N.B., Schwartz, W., Wronecki, A.J. and Vejborg, I. (2017) Outcome of Breast Cancer Screening in Denmark. BMC Cancer, 17, 897.

[4] Liberman, L., Gougoutas, C.A., Zakowski, M.F., et al. (2001) Calcifications Highly Suggestive of Malignancy: Comparison of Breast Biopsy Methods. American Journal of Roentgenology, 177, 165-172.

[5] Philpotts, L.E., Shaheen, N.A., Carter, D., Lange, R.C. and Lee, C.H. (1999) Comparison of Rebiopsy Rates after Sterotactic Core Needle Biopsy of the Breast with 11-Gauge Vacuum Suction Probe versus 14-Gauge Needle and Automatic Gun. American Journal of Roentgenology, 172, 683-687.

[6] Maxwell, A.J., Ridley, N.T., Rubin, G., Gilbert, F.J. and Michell, M.S. (2009) The Royal College of Radiologists Breast Group Breast Imaging Classification. Clinical Radiology, 64, 624-627. https://doi.org/10.1016/j.crad.2009.01.010

[7] Radiologi, Svensk Förening för Medicinsk. Klassifikation av Radiologiska Bilddiagnostiska Fynd i Bröst. Svensk Förening för Medicinsk Radiologi. http://www.sfmr.se/sidor/sfrb---rekommendationer/

[8] Bell, D., Weerakkody, Y., et al. (2016) Breast Imaging-Reporting and Data System (BI-RADS). Radiopedia.org. https://radiopaedia.org/articles/breast-imaging-reporting-and-data-system-birads

[9] Liberman, L. (2002) Percutaneous Image-Guided Core Breast Biopsy. Radiologic Clinics of North America, 40, 483-500.

[10] Sharma, E.S. (2014) Estimation for Lesion Depth in Mammograms Using Stereotactic Biopsy. International Journal of Advanced Research in Computer Science and Software Engineering, 4, No. 2.

http://ijarcsse.com/Before_August_2017/docs/papers/Volume_4/2_February2014/V 4I2-0181.pdf

[11] Nakamura, Y., Urashima, M., Mutsuura, M., Nishihara, R., Itoh, A., Kagemoto, M. and Hagiki, K. (2010) Stereotactic Directional Vacuum-Assisted Breast Biopsy using Lateral Approach. Breast Cancer, 17, 286-289.

[12] Sim, Y.T., Litherland, J., Lindsay, E., Hendry, P., Brauer, K., Dobson, H., Cordiner, C., Galiardi, T. and Smart, L. (2015) Upgrade of Ductal Carcinoma in Situ on Core Biopsies to Invasive Disease at Final Surgery: A Retrospective Review across the 
Scottish Breast Screening Programme. Clinical Radiology, 70, 502-506.

[13] Sinn, H.P. and Kreipe, H. (2013) A Brief Overview of the WHO Classification of Breast Tumors. Breast Care, 8, 149-154.

[14] WHO (2012) Classification of Tumors of the Breast. 4th Edition.

[15] Kettritz, U., Morack, G. and Decker, T. (2005) Stereotactic Vacuum-Assisted Breast Biopsies in 500 Women with Microcalcifications: Radiological and Pathological Correlations. European Journal of Radiology, 55, 270-276.

[16] Kettritz, U., Rotter, K., Schreer, I., Murauer, M., Schulz-Wendtland, R., Peter, D. and Heywang-Köhbrunner, S.H. (2004) Stereotactic Vacuum-Assisted Breast Biopsy in 2874 Patients-A Multicenter Study. Cancer, 100, 245-251. https://doi.org/10.1002/cncr.11887

[17] Uematsu, T., Kasami, M. and Yuen, S. (2008) Usefulness and Limitations of the Japan Mammography Guidelines for the Categorization of Microcalcifications. Breast Cancer, 15, 291-297. https://doi.org/10.1007/s12282-008-0033-4

[18] Liberman, L., Abrahamson, A.F., Squires, F.B., Morris, E.A. and Dershaw, D.D. (1998) The Breast Imaging Reporting and Data System: Positive Predictive Value of Mammographic Features and Final Assessment Categories. American Journal of Roentgenology, 171, 35-40.

[19] Mendez, A., Cabanillas, F., Echenique, M., et al. (2004) Mammographic Features and Correlation with Biopsy Findings using 11-Gauge Stereotactic Vacuum-Assisted Breast Biopsy (SVABB). Annals of Oncology, 15, 450-454.

[20] Sanders, M.A., Roland, L. and Sahoo, S. (2010) Clinical Implications of Subcategorizing BI-RADS 4 Breast Lesions Associated with Microcalcification: A Radiology-Pathology Correlation Study. The Breast Journal, 16, 28-31.

[21] Steger, H.E. and Pape, C. (1972) Beitrag zur Feinstruktur der sogenannten Mikrokalzifikation in Mamma tumoren. Zentralblatt Fur Allgemeine Pathologie, 115, 106.

[22] EUSOMA (European Society of Breast Cancer Specialists). https://www.eusoma.org/

[23] Chang, J.H., Vines, E., Bertsch, H., et al. (2001) The Impact of a Multidisciplinary Breast Cancer Center on Recommendations for Patient Management: The University of Pennsylvania Experience. Cancer, 91, 1231-1237. https://www.ncbi.nlm.nih.gov/pubmed/11283921

[24] Wiley, E.L. and Keh, P. (1999) Diagnostic Discrepancies in Breast Specimens Subjected to Gross Reexamination. The American Journal of Surgical Pathology, 23, 876-879. https://www.ncbi.nlm.nih.gov/pubmed/10435555

[25] Hansen, R.P., Vested, P., et al. (2011) Time Intervals from First Symptoms to Treatment of Cancer: A Cohort Study of 2212 Newly Diagnosed Cancer Patients. BMC Health Services Research, 11, 284. 